\title{
Use of small bowel capsule endoscopy in clinical practice: how has it performed?
}

Seong Ran Jeon

Digestive Disease Center, Institute for Digestive Research, Soonchunhyang University College of Medicine, Seoul, Korea

Received: June 12, 2020 Accepted: June 12, 2020

\section{Correspondence to}

\section{Seong Ran Jeon, M.D.}

Digestive Disease Center, Institute for Digestive

Research, Soonchunhyang University College of Medicine, 59 Daesagwan-ro, Yongsan-gu, Seoul 04401, Korea

Tel: +82-2-709-9202

Fax: +82-2-709-9696

E-mail: 94jsr@hanmail.net https://orcid.org/0000-00016970-9737

\section{See Article on Page 889-896}

Capsule endoscopy (CE) has remarkably changed the diagnostic approach to small bowel diseases. A number of clinical studies have evaluated the role of $\mathrm{CE}$ in obscure gastrointestinal bleeding (OGIB), Crohn's disease, and enteropathy/enteritis due to the variety of causes, including nonsteroidal anti-inflammatory drug. CE is currently recommended as the first-line procedure in OGIB patients [1]. It is the most sensitive diagnostic examination for detecting mucosal lesions of the small bowel in suspected Crohn's disease (sCD) or established CD (eCD) patients [1]. Due to misdiagnosis causing errors or delays of patient treatment, appropriate diagnosis using CE is crucial.

In the current issue of the Korean Journal of Internal Medicine, Kim et al. [2] investigated the time trend of practice patterns in using CE based on 17 years of nationwide registry data in Korea. Authors compared data from the previous 12 years (October 2002 to August 2014) to the recent 5 years (September 2014 to April 2019). The report was concluded with three observations. First, OGIB was consistently the primary indication for $\mathrm{CE}$, regardless of the time period. Second, the incomplete procedure rate of $\mathrm{CE}$ has de- creased over time. Third, inadequate bowel preparation was significantly associated with capsule retention and incomplete examination.

OGIB is consistently the primary indication for small bowel endoscopy $[3,4]$. The result of the present study suggested a similar result [2]. The most common indication for CE was the evaluation of OGIB (62.7\% vs. 68.8\%) during each period but had an increasing role in $\mathrm{CD}$ (3.5\% vs. $7.5 \%$ ) in the recent period. Until 2014, large numbers of patients with suspected small bowel diseases underwent diagnosis by radiologic modalities due to the considerably lower cost compared to $\mathrm{CE}$ in Korea. In the present study, the specific cutoff date for both periods was chosen, due to the expanded and partial coverage of health insurance being provided to OGIB and CD, respectively, since September 2014 [2]. Additionally, the therapeutic goal in patients with CD has been shifted to mucosal healing. The technological advanced CE system and radiological modalities induced an increasing trend of CE use in CD. The study by Kim et al. [2] reflects this trend and it demonstrated that CE use in CD significantly increased in the recent period.

In Asia, the incidence and prevalence of $\mathrm{CD}$ are rapidly increasing [5]. Although the use of CE in CD has 
increased, CE is not routinely used for diagnosis and evaluation of CD in Korea. The major complication of $\mathrm{CE}$ is capsule retention. Concerns of capsule retention in patients with CD lead to hesitation for performing $\mathrm{CE}$ in the real practice. According to previous studies, the capsule retention rate is reportedly between $1.3 \%$ and $2.5 \%$ [6]. In an analysis of 2,914 CE procedures for 10 years using nationwide CE registry data of Korea conducted in same study group in 2015, the capsule retention rate was $3 \%$ [3]. CE has a good safety profile; however, capsule retention in patients with $\mathrm{SCD}$ and eCD has increased by approximately 3.6\% and 10.4\%, respectively $[3,6-8]$. In a recent meta-analysis on capsule retention in $\mathrm{CD}$, the retention rates in $\mathrm{SCD}$ and eCD were $2.4 \%$ and $4.6 \%$, respectively [ 9 ]. In particular, the retention rate in eCD was considerably lower than the previously reported rate. The meta-analysis showed that the retention rate in eCD decreased after introducing patency capsule (PC) and radiological modalities such as magnetic resonance/computed tomography enterography (MRE/CTE) [9]. In a recent guideline, CTE/ MRE is recommended prior to $C E$ in patients with $C D$ [1], due to PC not being generally available in Korea. Although the study by Kim et al. [2] did not evaluate whether the use of MRE/CTE is associated with a decrease of capsule retention in $\mathrm{CD}$, the capsule retention rate was slightly lower (2.6\%) than that reported in the previous period (3.2\%). This can be interpreted in line with the results of previous research [9].

The results of the aforementioned study revealed that the incomplete rate of recent CE procedure (9.4\%) significantly decreased compared with that of previous CE procedures (18.9\%) [2]. The authors mentioned that the decreased incompleteness of recent CE may be due to the improvement of CE systems and radiological modalities. Risk factors leading to the incomplete rate of CE include old age, male, small bowel disease such as $\mathrm{CD}$ and tumor, and poor bowel preparation $[3,10]$. Adequate bowel preparation and complete procedure of CE play important roles to enhance diagnostic yield of CE. Kim et al. [2] also demonstrated that inadequate bowel preparation was significantly associated with incomplete procedure and capsule retention. This result is not surprising. Increasing indications, such as CD and tumors other than OGIB and small bowel strictures in these diseases interconnect inadequate bowel preparation, incomplete procedure and capsule retention.

Many studies using registry data, including the study by Kim et al. [2], have limitations such as selection bias, under-reporting or insufficient data, short observation periods, and a lack of long-term follow-up data. Therefore, a detailed analysis could not be performed. Nevertheless, the present study reflects the performance of $\mathrm{CE}$ in the real practice across 17 years since CE was first introduced in Korea. If more detailed/high qualified and long-term follow-up data can be produced in the nationwide CE registry; for example, analysis of capsule retention after use in CTE/MRE or PC and clinical impact of $\mathrm{CE}$ will be possible in the near future.

\section{Conflict of interest}

No potential conflict of interest relevant to this article was reported.

\section{Acknowledgments}

This work was supported by a Soonchunhyang University Research Fund.

\section{REFERENCES}

1. Shim KN, Jeon SR, Jang HJ, et al. Quality indicators for small bowel capsule endoscopy. Clin Endosc 2017;50:148160.

2. Kim SH, Lim YJ, Park J, et al. Changes in performance of small bowel capsule endoscopy based on nationwide data from a Korean Capsule Endoscopy Registry. Korean J Intern Med 2020;35:889-896.

3. Lim YJ, Lee OY, Jeen YT, et al. Indications for detection, completion, and retention rates of small bowel capsule endoscopy based on the 10-year data from the Korean Capsule Endoscopy Registry. Clin Endosc 2015;48:399-404.

4. Jeon SR, Kim JO, Kim HG, et al. Changes over time in indications, diagnostic yield, and clinical effects of double-balloon enteroscopy. Clin Gastroenterol Hepato 2012;10:1152-1156.

5. Ooi CJ, Hilmi I, Banerjee R, et al. Best practices on immunomodulators and biologic agents for ulcerative colitis and Crohn's disease in Asia. Intest Res 2019;17:285-310.

6. Liao Z, Gao R, Xu C, Li ZS. Indications and detection, completion, and retention rates of small-bowel capsule 
endoscopy: a systematic review. Gastrointest Endosc 2010;71:280-286.

7. Rezapour M, Amadi C, Gerson LB. Retention associated with video capsule endoscopy: systematic review and meta-analysis. Gastrointest Endosc 2017;85:1157-1168.e2.

8. Kim Y, Jeon SR, Choi SM, et al. Practice patterns and clinical significance of use of capsule endoscopy in suspected and established Crohn's disease. Intest Res 2017;15:467-
474.

9. Pasha SF, Pennazio M, Rondonotti E, et al. Capsule retention in Crohn's disease: a meta-analysis. Inflamm Bowel Dis 2020;26:33-42.

10. Westerhof J, Weersma RK, Koornstra JJ. Risk factors for incomplete small-bowel capsule endoscopy. Gastrointest Endosc 2009;69:74-80. 